\title{
Enterobacteriaceae y Staphylococcus Aureus en la etiologia de gastroenteritis infantiles
}

\author{
DRES, R. VIRGILIO *, A. M. CORDANO *, V. ESCOBEDO** y. E. ESCOBEDO*
}

INT RODUCción. Los sindromes diarreicos constituyen en el niño, una seria amenaza para su desarrollo y aún para su vida, ya que no es raro que produzcan profundas alteraciones del equilibrio ácido-básíco, de recuperación a veces larga y difícil. Es muy probable, que el origen bacteriano sea el más importante en su etiología; es por esto que en nuestro país se han dedicado numerosos estudios a determinar las especies bacterianas que con mayor frecuencia provocan estos sindromes $(1,2,6,10,11,12,13,17,20,21,22,23)$. Siempre queda un porcentaje relativamente alto de casos en que no se descubre ningún germen al que pueda atribuirse el cuadro observado $(1,17)$; esto podría deberse a que el verdadero causante esté fuera del campo de la investigación (parásitos, virus), a deficiencias técnicas (medios de cultivo inapropiados, inoportunidad en la toma de las muestras, posible efecto de algún tratamiento antibiótico previo) o a que el orígen de la diarrea está en transgresiones de la dieta o en factores extraintestinales.

Aunque, como ya se ha dicho, el tema ha sido abordado repetidamente, siempre se hace necesario ocuparse de la tiología de estos cuadros y tratar de evitar su aparición, por las consecuencias que pueda ocasionar en la salud del niño. Por otra parte, las especies bacterianas incriminadas pueden variar de un an̆o a otro, de manera que los resultados obtenidos en un determinado estudio pueden no ser necesariamente los mismos en otro período.

Hemos encontrado con alguna frecuencia Staphylococcus aureus en alimentos que provocaron intoxicación por contener algunas de las enterotoxinas estafilocócicas; dada la difusión que ha alcanzado este gếrmen en la población $(24,27,29)$

* Instíturo Bacieriologtco de Chile.

** Consultorio Andes S. N. S. hemos pensado que al proliferar en el intestino, $S$. aureus podría ser el origen de alguno de estos cuadros en el niõo.

En esta publicación se da cuenta de las investigaciones sobre la etiología de diarreas infantiles que realizamos entre Enero y Abril de 1972; en esta investigación además de las habituales Enterobacteriaceae consideradas como patógenas se incluyó la búsqueda de $S$ aureus.

Material Y Mítodos. Se tomaron muestras de deposición a 94 niños afectados de diarrea, 50 varones y 44 niñas, atendidos en el Consultorio Andes (Area Occidente de Santiago, Servicio Nacional de Salud). De ellos, 23 eran lactantes de 1 a 3 meses, 45 de 4 a 11 meses, 17 de 1 a 2 años y 9 mayores de 2 años. El estado nutritivo, evaluado personalmente por uno de los autores, sc consideró satisfactorio en la mayoría de los niños ya que solamente 20 presentaban un estado nutritivo deficiente, 10 grado I, 6 grado II y 4 grado III. La mayoría de los casos no había tenido tratamiento previo. Como control se tomaron muestras de deposición a un grupo análogo de 60 niños sanos, atendidos en el mismo consultorio y que viven en similares condiciones ambientales que los enfermos.

Muesstras: Las muestras se tomaron con tórula rectal a fin de evitar posibles contaminaciones externas. Esto fue hecho personalmente por uno de los autores, tanto en el grupo de los enfermos como en el de control. Las tórulas se prepararon en un alambre dentro de un catéter de goma de $6 \mathrm{~cm}$. de largo y $6 \mathrm{~mm}$. de diámetro; el otro extremo del alambre va tijo al tapón de corcho de un tubo; el conjunto (tubo con tórula y catéter) se esteritizó al autoclave. El catéter se introdujo en el recto, por el que se pasó la tórula de algodón y se frotó contra le pared del intestino. Las 
fórulas se volvieron al tubo esteril en que se prepararon, se cnviaron al Laboratorio y fucron sembradas en un lapso no mayor de 5 horas.

Cultivo: Se preparó una suspensión del material fecal agregando $1 \mathrm{ml}$. de caldo peptonado a cada tubo con tó ula, agitándola hasta obtener una suspensión homogénea. De esta suspensión se sembró $0,2 \mathrm{ml}$. en una placa de Wilson y Blair, $0,1 \mathrm{ml}$. en una placa de $S S$ y $0,1 \mathrm{ml}$. en una placa de Chapman; la siembra se extendió uniformemente en las placas con un rastrillo de vidrio estéril. Con lo que queda en el rastrillo se sembró una placa de MacConkey. Las placas se incubaron a $37^{\circ} \mathrm{C}$ y se examinaron a las 24 y 48 horas. Se sembró también un caldo de enriquecimiento según fórmula de Hajna (ver en 14). De este caldo se resembró a las 24 horas a placa de MacConkey. Todos los medios de cultivos empleados fueron preparados en el Instituto Bacteriológico.

Las colonias sospechosas de Salmonella o Shigella fueron aisladas y se sometieron a las pruebas habituales de identificación $(14,16)$. Escherichia coli enteropatógenos (ECEP) se pesquisaron por inmunofluorescencia (18, 19). Utilizamos sueros fluorescentes Difco, Pon! A y B, sueros fluorescentes monovalentes 026, 086, 0119, 0124, 0125, 0126,0127 Difco y 0111,055 y 0128 preparados por el Instituto Bacteriológico,

En las placas de Chapman se contaron las colonias con caracteres de $S$. aureaus y se catalogaron en cuatro grupos:

1. $\longrightarrow$ Negativas, las que tenían menos de 10 colonias/placa.

2.- Las que tenían entre 10 y 50.

3.-- Las que tenian entre 51 y $150 \mathrm{y}$,

4.- - las que tenían más de $\mathbf{1 5 0 .}$

Colonias representativas fucron repicadas a placas de agar sangre de conejo al $5 \%$ y su diagnóstico bacteriológico se confirmó por métodos microscópicos y bioquímicos $(3,4)$. Tres cepas de $S$. aurens aisladas de niños enfermos y tres aisladas de niños sanos fueron enviadas al Central Public Health Laboratory, Colindale, Londres, para determinar su fagotipo y eventual producción de enterotoxinas. Junto con estas cepas se enviaron también otras cuatro cepas aisladas anteriormente de alimentos que habían provocado intoxicación alimentaria.

Resultados. En 39 de las 94 muestras de deposición $(41,5 \%)$ de los nińos enfermos se aisló algunos de los gémenes investigados. En el grupo control de 60 niños sanos, 15 muestras fueron positivas $(25 \%)$. El detalle de estos resultados se expone en la tabla 1.
TABLA 1

SALMONELLA, SHIGELLA, ESCHERICHLA COLI ENTEROPATOGENO Y STAPHYLOCOCCUS AU. REUS ENCONTRADOS EN 94 DEPOSICIONES DE NIN̄OS CON DIARREA Y EN 60 DE NIÑOS SANOS

\begin{tabular}{|c|c|c|c|c|}
\hline Especies y serotipos & $\begin{array}{l}\text { Niños enf } \\
\text { No cepas }\end{array}$ & $\underset{\%}{e r m o s}$ & $\begin{array}{l}\text { Niños sa } \\
N^{o} \text { cepa }\end{array}$ & $\begin{array}{l}\text { anos } \\
\text { is \% }\end{array}$ \\
\hline Salmonelia & 5 & 5,3 & 0 & 0 \\
\hline S. newington & 2 & & & \\
\hline S. anatum & 1 & & & \\
\hline S. typhimuriam & 1 & & & \\
\hline S. derby & 1 & & & \\
\hline Shigella flexnerii & 2 & 2,1 & 0 & 0 \\
\hline E. colt enteropatógeno & 18 & 19,2 & 2 & 3,3 \\
\hline 055 & 4 & & 1 & \\
\hline 0111 & 3 & & & \\
\hline 086 & 3 & & & \\
\hline 0119 & 3 & & & \\
\hline 0124 & 2 & & & \\
\hline 0125 & 1 & & & \\
\hline 0127 & 1 & & & \\
\hline 026 & 1 & & 1 & \\
\hline $\begin{array}{l}\text { Staphylococcus aureus } \\
\text { más de } 151 \mathrm{col} / \text { placa * } \\
\text { entre } 51.150\end{array}$ & 21 & 22,4 & $1^{13}$ & 21,7 \\
\hline $\begin{array}{l}\text { col. /placa } \\
\text { entre } 10.50\end{array}$ & 11 & & 5 & \\
\hline col./place & 5 & & 7 & \\
\hline
\end{tabular}

* Cada tórula con muestra se suspendió en $1 \mathrm{ml}$. de caldo peptonado; de esta suspensión se sembró $0,1 \mathrm{ml}$. en placa de Chapman.

En total fueron 46 las cepas aistadas en las 39 muestras positivas, to cual se explica porque en 7 de estas muestras se encontraron dos de las especies investigadas; en 2 muestras $S$. aureus estuvo asociado a Salmonella y en atras 2 a ECEP; en una se encontró Salmonella junto con ECEP y en las dos últimas muestras hubo dos serotipos distintos de $E C E P$.

Discusión. Los niños que han sido objeto de este estudio presentaron todos una sintomatología intestinal relativamente moderada, tanto desde el punto de vista funcional como en su repercusión sobre el estado general. La mayoría tuvo un número de deposiciones que fluctuó entre cuatro a ocho diarias. Sólo uno debió ser hospitalizado por presentax una marcada deshidratación; 9 casos presentaron fiebre y 13 tuvieron vónitos. La evolución fue en general favorable, respondiendo rápidamente (en 2 a 3 días) al tratamiento antibiótico o simplemente dietético. Las deposiciones fueron de consistencia líquida, de color amarillo o 
verdoso con o sin presencia de mucus. Sólo en 8 se obscrvó sangre.

Los cinco casos en que se encontró Salmonella no se diferenciaron grandemente del resto del grupo; un caso con $S$. typhimurium presentó la diarrea más intensa, con una frecuencia de hasta 10 deposiciones diarias; sometido a tratamiento con Cloramfenicol (CAF) mejoró rápidamente. En una niña de 5 meses se aisló $S$. derby después del séptimo día de diarrea y sexto de estar en tratamiento con Neomicina. Mejoró con dietética y cambio a otro medicamento. De 2 casos son $S$. negwington uno presentó mucus y el otro mucus y sangre en la deposición; este último tenía dos días de evolución y 24 horats de tratamiento con CAF; se continuó el tratamicnto con ese mismo antibiótico y el niño mejoró en dos a tres días. Lo curioso es que esta cepa de $S$. newington "in vitro" resultó resistente al CAF. En todos estos casos el cuadro fue solamente local, señalando que estas salmonelosis pueden manifestarsc en forma benigna.

Las Salmonellas se están descubriendo actualmente como agentes importantes de gastroenteritis infantil (GEI) $(9,17)$. En el $V$ Congreso Latinoamericano de Punta del Este, Uruguay 1971, se describe en Brasil un $4,5 \%$ de diarreas como causadas por Salmonella (Resúmenes del Congreso, $\left.\mathrm{G}_{1}\right)$. En Argentina se ha apreciado un iumento de esta etiología de 6 a $30 \%$ en los últimos años. Especial importancia se está concediendo a $S$. typhimurium que suele encontrarse en alta incidencia en estos cuadros diarreicos (ibid $\mathrm{G}_{3}$, $\left.\mathrm{G}_{4}\right)$, provoca brotes epidémicos y ocasiona serios problemas por infección intrahospitalaria (ibid $\mathrm{G}_{5}$, $\left.G_{6}, G_{i}\right)$. En el presente cstudjo encontranos una frecuencia más alta de Salmonella grupo E que de $S$. typhimurium ya que de cinco Salmonella aisladas, 3 son del grupo E ( 2 s, newington, 1 $S$. anaium) y sólo 1 es $S$. typhimurium. Estos resultados concuerdan con los que obtuvinos en otro estudio nuestro (9) que abarca un período de un año y un muestreo más amplio en el número y origen de las cepas, ya que en cllos se observa un marcado predominio de $S$. newington sobre el resto de los serotipos.

En cuanto a Shigellosis, encontramos sólo dos casos por $S$. flexnerii $(2,1 \%)$ con una incidencia semejante a la descrita en Brasil $(2,4 \%$ ) (ibid, $\left.\mathbf{G}_{1}\right)$. Uno de estos casos correspondió a una niña de 14 meses con desnutrición grado II. En la tarde del dia de la consulta y toma de la muestra se agravó, siendo necesario cnviarla al hospital, en donde recibió hidratación parenteral. La sintomatología intestínal demoró 5 a 6 días en desaparecer. Un coprocultivo hecho durante la hospitalización fue negativo.

En 1945 la incidencia de cepas de Shigella aisladas en el Instituto Bacteriológico (6) fue cuatro veces superior a la de Salmonella; otras publicaciones anteriores a $1960(10,22)$ también hacen referencia a diarrea ocasionada principalmente por Shigella. Es posible que Shigella como agente de GEI haya disminuído considerablemente entre nosotros.

Se encontró ECEP en el 19,2\% de los niños con diarrea, las cifras que dan diversos autores para casos equivalentes varían entre 7,8 y $23 \%$ $(22,1)$. En el país no conocemos otro trabajo que cl de Meléndez y col. (20) en que se haya investigado ECEP por inmunofluorescencia; ellos dan una cifra muy alta, $45,2 \%$ de resultados positivos, en un número pequeño de 53 lactantes hospitalizados en un mismo hospital; quizás si esta circunstancia haya operado una selección de casos que explique la discordancia. Nuestro $19,2 \%$ encontrado en los niños enfermos contrasta con el $3,3 \%$ de los niños sanos.

Es conocido el hecho que $S$. aureas cs capaz de elaborar enterotoxinas (ET) muy activas $(7,25)$. En nuestro laboratorio hemos logrado identificar $S$. aureus como agente productor de intoxicaciones alimentarias de diversa intensidad (datos no publicados). Diversos autores han descrito casos de gastroenteritis en adultos, atribuídos a pululación intestinal de $S$. aureus $(5,8,15,26$, 28 ); todos ellos atribuyen la diarrea a canbios en el equilibrio de la flora intestinal provocados por tratamicntos antibiótico, que al inhibir la multiplicación de las bacterias sensibles, permite el desartollo exagerado de estafilococcus patógenos y resistentes. Nosotros creímos posible que en el organismo más delicado de los niños, cuadros dc GEI fueran debidos a $S$. aureus que aún sin tratamiento antibiótico previo, pudieran desarrollarse cn el intestino y actuaran por medio de una ET.

La investigación que hicimos nos dio un alto porcentaje de muestras con gran cantidad de $S$. aureus en niños que en su mayoría $(18 / 21)$, no habían recibido tratamiento antibiótico. El porcentaje $(22,4 \%)$ es en realidad mayor que cl dc cualquiera de los otros grupos de gérmenes considerados patógenos (Salmonella, Shigella, ECEP). Cierto es que en el grupo de niños sanos se encontró $S$, aureus en un porcentaje análogo $(21,7 \%)$; sin embargo, el recuento de colonias hecho en condiciones estandarizađas (véase métodos), es más alto en los niños enfermos que en los sanos. Así de los 21 niños enfermos en los que se encontró $S$. aureus $16(76 \%)$ tenían más de 51 colonias por placa, mientras que en los 13 niños sanos, apenas 6 presentaron recuentos superiores a 51 colonias por placa $(46 \%)$ y sólo uno de éstos tenía más de 150 colonias por placa (ver tabla 1). En un pequeño número de cepas se determinó el fagotjpo y producción de ET (tabla 2). De gran importancia nos parece el hecho que liss cepas aisladas de las deposiciones de niños enfermos, fueron iguales a cepas aisladas anteriormen- 
te de alimentos que proyocaron intoxicaciones alimentarias. En contraste con estos resultados, tres cepas aisladas de deposiciones de niños sanos pertenccían a otro fagotipos $\mathrm{y}$ solamente una $\mathrm{d}$ z ellas producía ET de tipo A.

\section{T A B L A 2}

\begin{tabular}{|c|c|c|c|c|c|c|c|}
\hline \multirow[t]{2}{*}{ Fagotipo } & \multicolumn{4}{|c|}{ Enterotoxinas } & \multirow{2}{*}{$\begin{array}{l}\text { Niños' } \\
\text { enfer. }\end{array}$} & \multirow{2}{*}{$\begin{array}{l}\text { Alimen. } \\
\text { contam. }\end{array}$} & \multirow{2}{*}{$\begin{array}{l}\text { Niños } \\
\text { sanos }\end{array}$} \\
\hline & $A$ & $B$ & $C$ & & & & \\
\hline 187 & + & 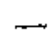 & + & - & 2 & 3 & 0 \\
\hline 81 & - & - & - & 一 & 1 & 1 & 0 \\
\hline $\begin{array}{l}\text { No de cepas } \\
\text { analizadas }\end{array}$ & & & & & 3 & 4 & 3 \\
\hline
\end{tabular}

Aunque el número de casos es muy reducido para sacar alguna conclusión, los datos expuestos permiten conjeturar que las cepas de $S$. aureus provenientes de niños con diarrea pudieron ser diferentes de las que se encuentran en niños sin diarrea; y que ciertas cepas de $S$. aureus capaces de colonizar el intestino y actuando por su ET sean la causa del cuadro diarreico.

\section{RESUMEN}

A 94 niños con diarrea, atendidos en el Consultorio Andes, se les tomó con tórula rectal y en el momento de la consulta, una muestra de deposición para practicar el correspondiente coprocultivo. El mismo examen se practicó a un segundo grupo de 60 niños sanos en ese mismo Consultorio. Del primer grupo, en $5.3 \%$ de los casos se aisló Salmonella; en 2,1 Shigella flexnerii; en $19,2 \%$ Escherichia coli enteropatógeno (ECEP); y en 22,4 Staphyloccoccus aureus. Ert el segundo grupo no se aisló Salmonella ni Shigella, sólo se encontró ECEP en $3,3 \%$ y S. aureus en $21,7 \%$. De los 5 casos de Salmonella encontrados, 2 fueron $\mathrm{S}$. newington, $I \mathrm{~S}$. austum, $I \mathrm{~S}$. tiphymurium y $1 \mathrm{~S}$. derby. La proporción de ECEP fue significativamente más alta en los niños entermos to de $\mathrm{S}$. aureus fue sensiblemente igual en los dos grupos $(22,4$ y $21,7 \%)$ pero el recuento de colonias por placa fue superior en el grupo de los niños enfermos. Un pequeño número de cepas de $\mathrm{S}$. aureus fue estudiado respecto a su fagotipo y a la capacidad de producir enterotoxinas. Cepas aisladas de niños enfermos demostraron ser idénticas en estos caracteres a otras cepas aistadas anteriormente de alimentos que provocaron intoxicaciones; en cambio, las cepas encontradas en niños sanos fueron completamente diferentes. Las Enterobacteriaceae consideradas patógenas se encontraron en 26,6\% de los nintos enfermos; si se agrega $\mathrm{S}$. aureus como posible causa de gastroenteritis se llega a $41,5 \%$.

\section{SUMMARY}

Rectal swabs were withdrawn from 94 Infants affectod summer diarrhea attending the Consultorio Andes of the National Heath Service and cultured. Salmonella were isolated from $5,3 \%$ of the swabs; Shigella from $2,1 \%$; enteropathogenic Escherichia coli (ECEP) from $19,2 \%$ and Staphylococcus aureus from $22,4 \%$. Three of the Salmonella atrains were found to be of group $\mathrm{E}$ (S. newington and S. anatum), $1 S$. typhimurium and 1 $S$. derby. The two Shigella were S. flexnerii. Analogous cultures were performed in a group of 60 normal infants; neither Salmonella nor Shigella were obtained in this group, but ECEP and S. aureus were recovered in 3,3\% and $21.7 \%$ respectively. ECEP was significantly higher in sick infants that in normal ones. A few Strains of $S$. aureus were phage-typed and analysed with respect to their enterotoxigenicity; strains from diseased infants were identical in these features to strains isolated from food poisoning ontbreaks; strains from normal infants were enterely different. Pathogenic Enterobacteriaceac were found in $26.6 \%$ of the sick infants; if $S$. aureus is considered as an ethiologic agent of diarrhea this percentiage rises to $41,5 \%$.

\section{BibLIOGRAPÍA}

1.-Aguilo, C., Rizzardini, M., Manubens, F. y Schusw ter, A. Tratamiento de la diarrea aguda grave del lactante con Gentamicina. Pediatría, 9: 74, 1966.

2,-Aguilo. C., Soriano, H., Guerrero, F. y Pino, C. Acido nalidíxico en el tratamiento de la diarrea aguda del lactante. Pediatría 11: 224-231. 1968.

3.-Buird-Parker, A. C. Recomendation of the Sub Committee on Staphylococci and Micrococci I.nter. Bull. Bact. Nomen. Taxon. 15: 109, 1966.

4.-Bairl-Parker, A. C. The Classification of Staphylococci and Micrococei from world-wide sources. J. Gən. Bacteriol. 38: 363-387, 1965.

5.-Borber, M. A. Garrod, L. F. Antibiotic and Chemotherapy. E. \& S. Livingstone Ltd., Edinburg and London, 1963.

6.- Canessa, E. y Garcés, C. Distribución por especies de Shigellas y Salmonellas clasificadas en la sección de gérmenes Entéricos. Rev. Ch. Ped. 16: 697-700. 1945.

7.-Cassman, E. P., Bevinett, R. W., Dorsey, A. E. \& Issa, J. A. Identification of a fourth Staphylococcal enterotoxin, enterotoxin D. J. Bacteriol. 90; 1481. 1967.

8.-Cook, 1., Elliott, C., Elliott-Smith, A., Frisby. B. R. \& Gardenr, A. M. N. Stapbylococcal diarrhoea. With an acount of two butbreaks in the same hospital. Brit. Med. J. 1: 542-547, 1957. 
9.-Cordano, A. M., Virgilio, R., y Escobedo, M. E. Salmonella grupo $\mathrm{E}$ aistadas en Chile. Rev, Lat. Amer. Microbjol, 15: 1-4, 1973.

10. - Cosia, $A$. La disentería bacilar en la infancia. Rev. Ch. Ped. 16: 646-661, 1945.

11.-Costa, A. y Arcaya, O. Escherichia coli patógeno en la diarrea del niño. Rey. Ch. Ped. 26: 451, 1955.

12.-Costa, A. y Arcaya, O. Escherichia coli patógeno en diarreas agudas y toxicosis del niño menor. Rev. Ch. Ped. 27: 233, 1956.

13.-Donoso, P., Montoya, C. y Contreras, J. Diarrea aguda por Shigella en el lactante. Rev. Ch. Ped. 35: $1151-1156,1964$.

14.-Edwards, $P . R$. \& Ewing, $W . N$. Identification of Entercbactariaceae Burgess Publishing Co., Minneapolis 15, Minnesota, U. S. A. 1972.

15.-Khan, M. T.\& Hall, W. H. Stapbylococcal enterocolitis. Treatment with oral Vancomycin. Ann. Int. Med. 65: 1-8, 1966.

16.- Le Minor, L. Le Diagnostic de Laboratoire des Enterobactéries 3e. édit. Editions de la Tourelle, Saint Mandé, 1969.

17.-Lobos, H., Aguilar, C. y Romero, H. Bacteriología de las diarreas agudas en el lactante hospitalizado. Pediatría 8: 280-293. 1965.

18.- Maraden, H. B., Hyde, W. A. \& Bracegirdle, E. Inmunofluorescence in the diagnosis of enteropathogenic Escherichia coli infeccions. Lancet $I$ : 189-191, 1965 .

19.-Martin, A. J. \& O'Brien. M. Detection of enterophatogenic Escherichia coli in fecal cultures by use of a modified flıorescent —antibady technique. J. Bacteriol, 89: 570-573, 1965.

20.-Meléndez, M., Greve, E., Cassoria, R., Lobos, $\boldsymbol{H}$. y Jacob, C. Diagnóstico de Escherichia coli enteropatógenas en diarreas infantiles por el método de inmunofluorescencia. Rev. Ch. Ped. 42: 169173, 1971.
21.- Prado, V, Donoso E,. y Zillenuelo, G. Infección enteral por Salmonella typhymurium en lactantes y la sensibilidad in vitro de este germen patógeno a siete antibióticos. Rev. Ch. Ped. 42: 91-96, 1971.

22.- Prado, E. y Diessert, E. Bacteriología de las diarreus en niños. Bol. Inst. Bacteriol. Ch. $X: 37-42$, 1957.

23.-Rodriguez, M., Meneghello, I. y Adasme, R. Estudios sobre la Escherichia coli II. Investigación de cepas 0111,055 y 026 en lactantes con entero. colitis aguda, toxicosis y en nifios sin sindrome diatreico, Rev. Ch. Ped, 27: 197. 1956

24.-Solberg, C. O. A study of carriers of Staphylococcus aureus. Acta Med. Scand. 778 (suppl) : 436, 1965.

25.-Smikovocova, M. \& Gilbert, $R$. $J$. Serological detection of Enterotoxin from food-poisoning strains of Staphylococcus aureus. J. Med. Microbiol. 4: 19-30, 1971.

26.- Surgalla. M. I. \& Dack, Gail M. Enterotoxin produced by Micrococci from cases of enteritis after antibiotic therapy. JAMA I58: 649, 1955.

27.-Virgilio, R., González, C., Mendoza, S., Avendaño, $S$. y Muñoz, N. Bacteriological Analyses of frozen Shrimp. 2. Staphylococci in Precooked Frozen Chilean Shrinip. J. Food Sci. 35: 845-848, 1970.

28.-Wallace, J. F., Smith, R., \& Peterdorf, R. Oral administration of Vancomicin in the treatment of Staphylococcal enterocolitis. N. Eng. J. Med. 272: 1014-1015, 1965.

29.-Williams, R. E. O. Healhy Carriage of Staphylococcus aureus; its prevalence and importance. Bacteriol, Rev. 27: 56-71, 1963.

\section{AGRADECIMIENTOS}

Expresamos nucstro agradecimiento al Dr, R. J. Gilbert del Central Public Health Lahoratory, Colindale, Londres, por su ineslimable cooperación eri la determinación de fagotipos $y^{\prime}$ producción de enterotoxinas de $s$. aureus. 